\title{
COMPLICAÇÕES NA SALA DE RECUPERAÇÃO ANESTÉSICA, FATORES DE RISCOS E INTERVENÇÕES DE ENFERMAGEM: REVISÃO INTEGRATIVA
}

\author{
Complications in the post-anesthesia care unit, risk factors \\ and nursing interventions: an integrative review
}

\author{
Complicaciones en la sala de recuperación anestésica, factores de riesgos \\ y intervenciones de enfermado: revisión integrativa
}

\author{
Mariângela Belmonte Ribeiro', Aparecida de Cassia Giani Peniche², Silvia Cristina Fürbringer e Silva ${ }^{3}$
}

RESUMO: Objetivos: Identificar as complicações e os riscos que os pacientes possam desenvolver no pós-operatório imediato e propor um instrumento que contenha as complicações, riscos e intervenções de enfermagem individualizadas. Método: Revisão integrativa da literatura realizada entre março e outubro de 2015 nas bases de dados PubMed (arquivo digital produzido pela National Library of Medicine) e Biblioteca Virtual em Saúde (BVS). Para o levantamento dos artigos foram utilizados os descritores: sala de recuperação, complicações e cuidados de enfermagem. Resultados: A estratégia de busca permitiu a análise de 15 artigos. As complicações, riscos e intervenções foram identificadas e assim categorizadas: hipotermia, hipoxemia, apneia; edema agudo de pulmão, tremores, náuseas e vômitos; retenção urinária, grau de dependência de cuidados; disritmias cardíacas, complicações gerais; complicações com idosos e posicionamento cirúrgico. Conclusão: As complicações e riscos levantados, assim como as intervenções, foram a base para a construção do instrumento. Palavras-chave: Sala de recuperação. Complicações intraoperatórias. Cuidados de enfermagem.

ABSTRACT: Objectives: To identify complications and risks that patients can develop in the immediate postoperative period and to propose an instrument that addresses the complications, risks and individualized nursing interventions. Methods: We conducted an integrative review of the literature between March and October 2015 in PubMed databases (digital archive produced by the National Library of Medicine) and Virtual Health Library (Biblioteca Virtual em Saúde - BVS). We used the following descriptors in our search: recovery room/sala de recuperação/sala recuperación; postoperative complications/complicações pós-operatórias/complicaciones pós-operatórias; and nursing care/cuidados de enfermagem/atención de enfermeira. Results: The search strategy allowed the analysis of 15 articles. Complications, risks and interventions were identified and categorized as follows: hypothermia, hypoxemia and apnea; acute pulmonary edema, tremors, nausea and vomiting; urinary retention and degree of dependence on care; cardiac dysrhythmias and general complications; complications in the elderly and surgical positioning. Conclusion: The resulting complications, risks and interventions were the basis for the construction of our instrument. Keywords: Post-anesthesia care unit. Intraoperative complications. Nursing care

RESUMEN: Objetivos: Identificar las complicaciones y los riesgos que los pacientes pueden desarrollar en el período posoperatorio inmediato y proponer un instrumento que aborde las complicaciones, los riesgos y las intervenciones individualizadas de enfermería. Métodos: Realizamos una revisión integrativa de la literatura entre marzo y octubre de 2015 en las bases de datos de PubMed (archivo digital producido por la Biblioteca Nacional de Medicina) y la Biblioteca Virtual en Salud (BVS). Utilizamos los siguientes descriptores en nuestra búsqueda: recovery room/sala de recuperação/sala de recuperación; postoperative complications/complicações pós-operatórias/ complicaciones pós-operatórias; y nursing care/cuidados de enfermagem/atención de enfermería. Resultados: La estrategia de búsqueda permitió el análisis de 15 artículos. Las complicaciones, los riesgos y las intervenciones se identificaron y categorizaron de la siguiente manera: hipotermia, hipoxemia y apnea; edema agudo de pulmón, temblores, náuseas y vómitos; retención urinaria y grado de dependencia de la atención; disrritmias cardíacas y complicaciones generales; complicaciones en ancianos y posicionamiento quirúrgico. Conclusión: Las complicaciones, riesgos e intervenciones resultantes fueron la base para la construcción de nuestro instrumento. Palablas clave: Sala de recuperación. Complicaciones intraoperatorias. Atención de enfermería.

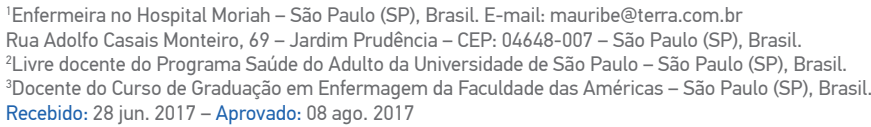




\section{INTRODUÇÃO}

A sala de recuperação anestésica (SRA) é o local destinado a receber os pacientes no pós-operatório imediato e tem como objetivo prevenir possíveis complicações decorrentes do ato anestésico cirúrgico. Para tanto, há necessidade de recursos humanos especializados e materiais diferenciados para prestar uma assistência de qualidade ${ }^{1}$.

A atuação da equipe de enfermagem é fundamental nesse período de instabilidade, no qual os riscos podem desencadear problemas, sendo de grande importância a estabilidade do nível de consciência e dos sinais vitais do paciente ${ }^{2}$.

A assistência de enfermagem nesse período deve ser planejada desde sua admissão até o paciente ter condições de alta da SRA. A sistematização da assistência de enfermagem é uma das estratégias para a obtenção de um processo seguro ${ }^{3}$.

Para a avaliação e o plano de cuidado do paciente na SRA algumas escalas são utilizadas, no entanto, muitas foram elaboradas e validadas por médicos, como a Escala de Aldrete e Kroulick ${ }^{4}$, entre outras. Essa escala é de 1970 e nos diais atuais ainda é a mais utilizada na SRA, porém, não avalia complicações ou riscos. Até o momento não existe um instrumento que atenda às necessidades dos cuidados de enfermagem, os quais são prestados em tempo integral ao paciente ${ }^{1}$.

Diante de tais considerações e da importância da segurança do paciente no pós-operatório imediato, compreendese que é necessário identificar as complicações e os riscos que aos quais os pacientes estão sujeitos e propor um instrumento que contenha as intervenções de enfermagem para cada complicação.

Assim, considera-se que uma revisão integrativa na temática das complicações na SRA se faz necessária para levantar os riscos e espera-se que este estudo possa contribuir para a prática profissional, por meio de levantamentos e evidências.

\section{OBJETIVOS}

Identificar as complicações e os riscos que os pacientes possam desenvolver na sala de recuperação anestésica.

Propor um instrumento que contenha as complicações, os riscos e as intervenções de enfermagem individualizadas.

\section{MÉTODO}

Para o levantamento das complicações e riscos na SRA e a construção do instrumento de avaliação, realizou-se uma revisão integrativa tendo como referencial metodológico seis etapas:

1. formulação do problema;

2. estabelecimento dos critérios de inclusão e exclusão;

3. levantamento de dados a serem extraídos dos textos.

4. avaliação dos dados coletados;

5. análise e interpretação dos dados; e

6. apresentação dos resultados ${ }^{5}$.

Para a formulação do problema, utilizou-se da seguinte pergunta norteadora: quais as complicações e riscos encontrados na literatura envolvendo os pacientes na SRA? O levantamento de dados ocorreu nos meses de março a outubro de 2016.

Foram selecionados os artigos nos idiomas português, inglês e castelhano indexados nas bases de dados PubMed (arquivo digital produzido pela National Library of Medicine) e na Biblioteca Virtual em Saúde (BVS), por disponibilizar um grande conteúdo de material científico, advindo das bases de dados referenciais nos campos da saúde. Os descritores utilizados foram: sala de recuperação and complicações and cuidados de enfermagem.

A busca de referências se iniciou em 1979. Esse período, alargado de 1979 a 2016, justificou-se pela escassa produção relacionada à SRA no que se refere à assistência de enfermagem prestada. Como critérios de inclusão foram utilizados artigos que relatavam complicações na SRA com pacientes adultos e como critério de exclusão artigos que relatavam complicações com crianças.

O fluxograma com o levantamento dos dados e o número de artigos que fizeram parte da amostra final está apresentado na Figura 1.

Para a coleta de dados foi elaborado um quadro com as seguintes informações: estudo, referência, base de dados ou portais, tipo de estudo, resultados e nível de evidências.

Para uma melhor classificação dos níveis de evidências dos artigos esses foi utilizada uma escala de I a VI ${ }^{6}$ da seguinte forma:

- nível 1: evidências resultantes da meta-análise de múltiplos estudos clínicos controlados e randomizados;

- nível 2: evidências obtidas em estudos individuais com delineamento experimental;

- nível 3: evidências de estudos quase-experimentais;

- nível 4: evidências de estudos descritivos (não experimentais) ou com abordagem qualitativa; 
- nível 5: evidências provenientes de relatos de caso ou de experiência;

- nível 6: evidências baseadas em opiniões de especialistas ${ }^{6}$.

Essa classificação é uma recomendação para os estudos de revisão, pois enriquece e fortalece as conclusões em relação ao tema pesquisado.

Os resultados são apresentados em uma tabela de forma descritiva, com as características e as implicações principais de cada estudo.

\section{RESULTADOS}

O Quadro 1 apresenta as principais características dos estudos selecionados na revisão integrativa.

Foram escolhidos 15 artigos que respondiam à questão da pesquisa e atendiam aos critérios de inclusão.
Entre os 15 artigos incluídos para avaliação das complicações pós-operatórias, verificou-se que 11 eram estudos de delineamentos observacionais transversais (nível de evidencia IV), 2 estudos de revisão (nível de evidencia V) e 2 estudos de caso (nível de evidencia V). Os anos de publicações mais frequentes foram os de 2010, com 4 publicações, e 2012, com 2. As outras foram entre os anos de 1979 e 2008.

Treze estudos foram publicados em periódicos português, um em periódico inglês e um em periódico espanhol.

A partir da análise crítica dos artigos, as complicações, riscos e intervenções foram identificadas e assim categorizadas: hipotermia, hipoxemia, apneia, edema agudo de pulmão, tremores, náuseas e vômitos, retenção urinária, grau de dependência de cuidados, disritmias cardíacas, complicações gerais e com idosos e posicionamento cirúrgico.

A pesquisa mostrou uma escassez de instrumentos para mensurar as complicações e os riscos pertinentes aos problemas que o paciente pode desenvolver nesse período, assim como

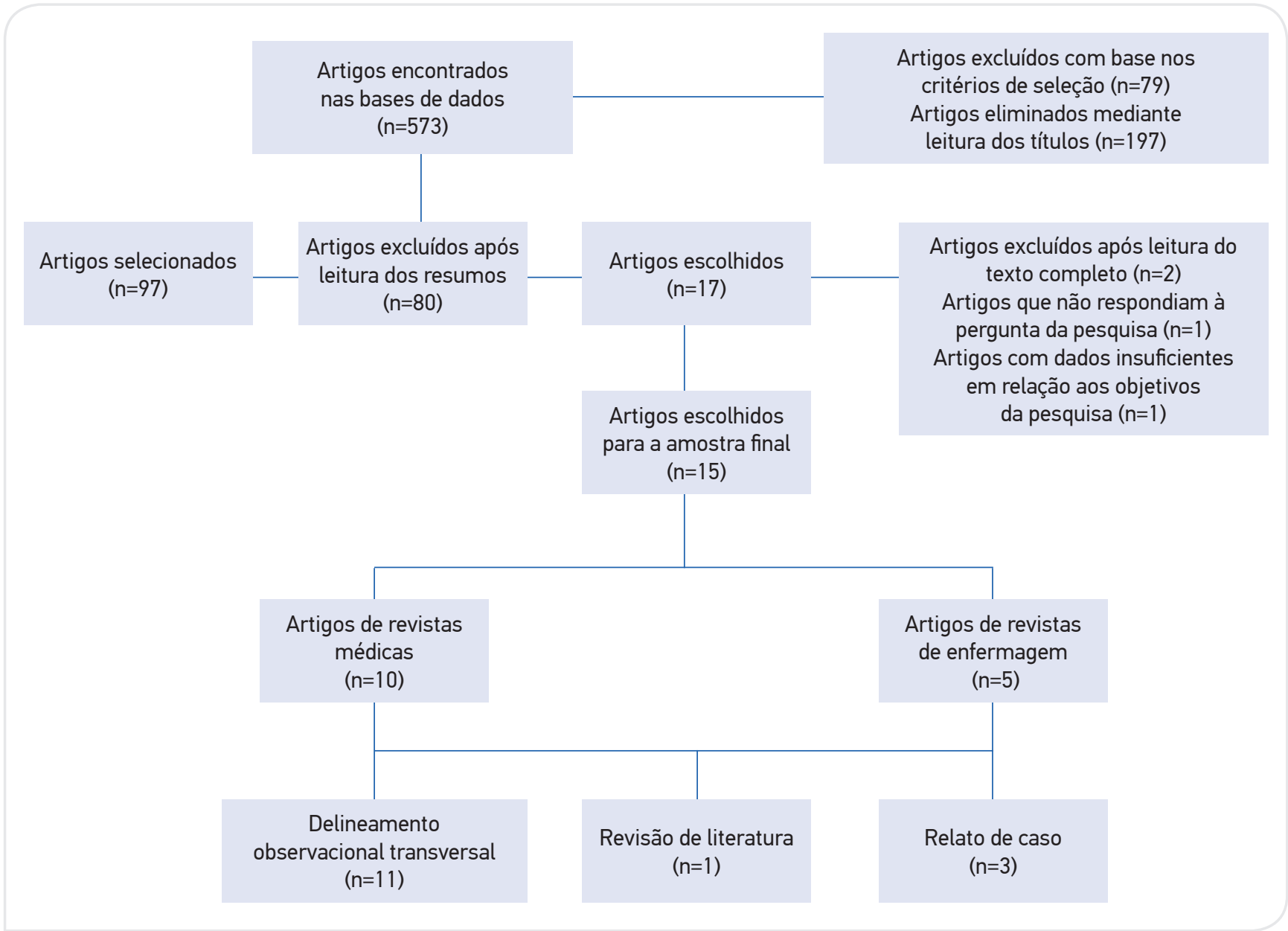

Figura 1. Fluxograma do processo de levantamento de artigos científicos. 
Quadro 1. Síntese dos estudos encontrados em relação às complicações na sala de recuperação anestésica.

\begin{tabular}{|c|c|c|c|c|c|c|}
\hline Estudo & Referência & $\begin{array}{l}\text { Base de } \\
\text { dados e } \\
\text { portais }\end{array}$ & Origem & $\begin{array}{l}\text { Tipo de } \\
\text { estudo }\end{array}$ & Resultados & $\begin{array}{l}\text { Nível de } \\
\text { evidência }\end{array}$ \\
\hline E1 & $\begin{array}{c}\text { Mattia et al. } \\
(2012)^{8}\end{array}$ & SciELO & Brasil & $\begin{array}{c}\text { Exploratório, } \\
\text { descritivo }\end{array}$ & $\begin{array}{l}\text { Os resultados demonstraram que } 80,0 \% \text { dos pacientes } \\
\text { mantiveram-se hipotérmicos até } 30 \text { minutos de } \\
\text { permanência na SRA, com temperatura axilar entre } \\
35,1 \text { e } 35,9^{\circ} \mathrm{C} \text {. A manifestação clínica de hipotermia foi } \\
\text { a hipoxemia, seguida de tremores, palidez cutânea e } \\
\text { hipertensão arterial. }\end{array}$ & IV \\
\hline E2 & $\begin{array}{l}\text { Zappelini } \\
\text { et al. } \\
(2008)^{10}\end{array}$ & LILACS & Brasil & $\begin{array}{c}\text { Analítico, } \\
\text { observacional }\end{array}$ & $\begin{array}{l}\text { A média de temperatura na SRA foi de } 34,5^{\circ} \mathrm{C} \text {. Os fatores } \\
\text { de risco foram: pacientes com } 60 \text { anos apresentaram } 0,5^{\circ} \mathrm{C} \\
\text { inferior em relação à média de temperatura na SRA; assim } \\
\text { como as cirurgias abdominais com tempo cirúrgico de } \\
180 \text { minutos; e a anestesia geral inalatória associada com } \\
\text { a raquianestesia, que apresentaram hipotermia na SRA. }\end{array}$ & IV \\
\hline E3 & $\begin{array}{l}\text { Amante } \\
\text { et al. } \\
(2012)^{9}\end{array}$ & LILACS & Brasil & Quantitativo & $\begin{array}{l}\text { As variáveis mensuradas foram: temperatura corporal } \\
\text { durante e imediatamente após a cirurgia; idade do paciente; } \\
\text { tempo, tipo de cirurgia e tipo de anestesia. Reafirma-se ser } \\
\text { necessária uma avaliação segura e eficaz da necessidade } \\
\text { de regulação térmica desses pacientes, de modo que } \\
\text { a intervenção na vigência de hipotermia perioperatória } \\
\text { diminua a incidência de complicações nesse período. }\end{array}$ & IV \\
\hline E4 & $\begin{array}{l}\text { Marcondes } \\
\text { et al. } \\
(2006)^{11}\end{array}$ & SciELO & Brasil & $\begin{array}{l}\text { Delineamento } \\
\text { observacional }\end{array}$ & $\begin{array}{l}\text { Houve maior incidência de hipoxemia moderada/intensa } \\
\text { durante o transporte de pacientes do sexo feminino e nos } \\
\text { pacientes estado físico ASA Il e III submetidos a cirurgias } \\
\text { cardiotorácicas, gastroproctológicas e de cabeça e pescoço. } \\
\text { A anestesia geral foi a maior incidência para hipoxemia. }\end{array}$ & IV \\
\hline E5 & $\begin{array}{l}\text { Oliveira } \\
\text { Filho et al. } \\
(2001)^{12}\end{array}$ & LILACS & Brasil & $\begin{array}{l}\text { Delineamento } \\
\text { observacional }\end{array}$ & $\begin{array}{l}\text { Quarenta e nove pacientes apresentaram } \mathrm{SpO}_{2} \text { menor } \\
\text { que } 92 \% \text { durante o período de observação. Fatores de } \\
\text { hipoxemia: idade maior que } 55 \text { anos, } \mathrm{SpO}_{2} \text { pré-operatória } \\
\text { menor que } 95 \% \text {, anestesia geral, hipoventilação detectada } \\
\text { clinicamente. A PAS e a FC foram significativamente mais } \\
\text { elevadas nos pacientes hipoxêmicos. }\end{array}$ & IV \\
\hline E6 & $\begin{array}{l}\text { Rezende } \\
(2003)^{15}\end{array}$ & SciELO & Brasil & $\begin{array}{l}\text { Relato de } \\
\text { caso }\end{array}$ & $\begin{array}{l}\text { A paciente foi extubada, obedeceu aos comandos } \\
\text { para respirar e colaborou na passagem à maca, sendo } \\
\text { transportada para a SRPA, aonde chegou consciente. } \\
\text { Minutos após, apresentou apneia, cianose e inconsciência. } \\
\text { Foi realizada ventilação manual com oxigênio a } 100 \% \text {, } \\
\text { seguida de injeção de naloxona ( } 0,2 \text { mg) por via venosa, } \\
\text { havendo retorno da ventilação espontânea e da consciência. }\end{array}$ & V \\
\hline E7 & $\begin{array}{l}\text { Bisinotto } \\
\text { et al. } \\
(2008)^{14}\end{array}$ & SciELO & Brasil & $\begin{array}{l}\text { Relato de } \\
\text { caso }\end{array}$ & $\begin{array}{l}\text { Após a extubação, a paciente apresentou laringoespasmo e } \\
\text { diminuição da saturação de oxigênio, foi estabilizada. Na SRA, } \\
\text { logo após a admissão, apresentou edema agudo de pulmão } \\
\text { com eliminação de secreção, recebeu suporte de oxigênio. }\end{array}$ & V \\
\hline E8 & $\begin{array}{l}\text { Mago et al. } \\
(2010)^{18}\end{array}$ & SciELO & Brasil & $\begin{array}{l}\text { Delineamento } \\
\text { observacional }\end{array}$ & $\begin{array}{l}\text { O critério para caracterizar retenção urinária foi: volume } \\
\text { vesical igual ou maior que } 600 \mathrm{~mL} \text {, associado à incapacidade } \\
\text { de micção espontânea } 30 \text { minutos após o diagnóstico. } \\
\text { Ocorreu retenção urinária em } 19 \text { pacientes. Os fatores } \\
\text { preditivos independentes identificados foram o volume } \\
\text { urinário igual ou maior que } 360 \mathrm{~mL} \text { na admissão à SRPA }\end{array}$ & IV \\
\hline E9 & $\begin{array}{l}\text { Albergaria } \\
\text { et al. } \\
(2007)^{16}\end{array}$ & SciELO & Brasil & $\begin{array}{l}\text { Artigo de } \\
\text { revisão }\end{array}$ & $\begin{array}{c}\text { Os tremores são, juntamente com náuseas e vômitos, } \\
\text { causas de intenso desconforto na SRA, além de } \\
\text { potencialmente prejudiciais por gerarem aumento da } \\
\text { demanda metabólica. }\end{array}$ & V \\
\hline
\end{tabular}


Quadro 1. Continuação.

\begin{tabular}{|c|c|c|c|c|c|c|}
\hline Estudo & Referência & $\begin{array}{l}\text { Base de } \\
\text { dados e } \\
\text { portais }\end{array}$ & Origem & $\begin{array}{l}\text { Tipo de } \\
\text { estudo }\end{array}$ & Resultados & $\begin{array}{l}\text { Nível de } \\
\text { evidência }\end{array}$ \\
\hline E10 & $\begin{array}{l}\text { Lages et al. } \\
\qquad(2005)^{17}\end{array}$ & SciELO & Brasil & $\begin{array}{l}\text { Artigo de } \\
\text { revisão }\end{array}$ & $\begin{array}{l}\text { Várias são as estratégias de manuseio de NVPO, } \\
\text { destacando-se, no entanto, as linhas de orientação } \\
\text { emitidas por Gan em 2003, sugere que o paciente com } \\
\text { história prévia de náuseas e vômitos sejam medicados } \\
\text { antes, durante e após a cirurgia. }\end{array}$ & V \\
\hline E11 & $\begin{array}{l}\text { Lima et al. } \\
(2010)^{1}\end{array}$ & SciELO & Brasil & $\begin{array}{l}\text { Transversal } \\
\text { prospectivo }\end{array}$ & $\begin{array}{c}\text { Os resultados apontam que os pacientes têm grau de } \\
\text { dependência entre intermediário e semi-intensivo. } \\
\text { Houve relação significativa entre grau de dependência e } \\
\text { classificação ASA. }\end{array}$ & IV \\
\hline E12 & $\begin{array}{l}\text { Nascimento } \\
\text { Júnior } \\
\text { et al. } \\
(2000)^{19}\end{array}$ & LILACS & Brasil & $\begin{array}{l}\text { Delineamento } \\
\text { observacional }\end{array}$ & $\begin{array}{l}\text { Realizada eletrocardiografia pré-operatória e na SRPA, } \\
33,6 \% \text { dos pacientes apresentaram disritmias, as mais } \\
\text { comuns foram taquicardia sinusal e bradicardia sinusal, } \\
\text { sendo que, na maioria das vezes, houve algum evento } \\
\text { prévio a essas alterações. A incidência dessas disritmias } \\
\text { foi equivalente em todos os grupos etários. }\end{array}$ & IV \\
\hline E13 & $\begin{array}{c}\text { Eltringham } \\
(1979)^{20}\end{array}$ & $\begin{array}{l}\text { PubMed/ } \\
\text { Medline }\end{array}$ & $\begin{array}{l}\text { Estados } \\
\text { Unidos }\end{array}$ & $\begin{array}{l}\text { Delineamento } \\
\text { observacional }\end{array}$ & $\begin{array}{l}\text { Os pacientes foram divididos em três grupos, de } \\
\text { acordo com a duração da sua permanência na SRA. } \\
\text { As complicações cardiovasculares estão divididas em } \\
\text { respiratórias e outras, embora alguns pacientes tenham } \\
\text { tido mais de uma complicação. }\end{array}$ & IV \\
\hline E14 & $\begin{array}{l}\text { Mendoza } \\
\text { e Peniche } \\
(2010)^{3}\end{array}$ & SciELO & Caribe & $\begin{array}{l}\text { Descritivo, } \\
\text { retrospectivo }\end{array}$ & $\begin{array}{l}\text { O sexo masculino foi predominante }(56,4 \%) \text {, hipertensos } \\
\text { (32,7\%) e foram classificados em ASA II (60\%). Quanto ao } \\
\text { intraoperatório, foram submetidos a cirurgia abdominal } \\
(53,6 \%) \text {, anestesia geral }(50,9 \%) \text {, posição de decúbito dorsal } \\
(81,8 \%) \text { e o tempo cirúrgico foi inferior a } 3 \text { horas (62,7\%). }\end{array}$ & IV \\
\hline E15 & $\begin{array}{l}\text { Popov e } \\
\text { Peniche } \\
(2009)^{2}\end{array}$ & SciELO & Brasil & $\begin{array}{l}\text { Exploratório, } \\
\text { retrospectivo }\end{array}$ & $\begin{array}{l}\text { As complicações prevalentes foram a dor e a hipotermia, } \\
\text { complicações que apresentaram relação estatisticamente } \\
\text { significante com as intervenções de enfermagem. }\end{array}$ & IV \\
\hline
\end{tabular}

SRA: sala de recuperação anestésica; PAS: pressão arterial sistêmica; FC: frequência cardíaca; NVPO: náuseas e vômitos pós-operatórios; ASA: American Society of Anesthesiologists.

os cuidados de enfermagem a serem realizados. Diante deste levantamento, uma escala com as complicações baseadas nas evidências dos artigos foi elaborada, com seus riscos pertinentes a cada obstáculo, assim como os cuidados de enfermagem. Os dados coletados submetidos à aprovação da análise estatística, para comprovação da viabilidade e autenticidade.

Foram identificadas 14 complicações associadas aos fatores de riscos que podem ocorrer em SRA. Na sequência, esses fatores foram transformados em perguntas simples e curtas para serem respondidas com sim ou não. Adotou-se que cada resposta "sim" equivaleria ao número 1 e cada resposta "não” equivaleria ao número 0.

A identificação do grau de risco, em baixo, médio e alto, de possíveis complicações na SRA foi obtida pela soma das respostas "sim" dadas ao questionário, a cada fator de risco que todo paciente estiver exposto.

Algumas complicações possuem apenas três fatores de risco, entretanto, outras têm nove. Dessa forma, a soma dos fatores de risco variou de 0 a 9 .
Assim, para a classificação do grau de risco, adotou-se uma escala numérica colorida e dividida em três partes iguais:

- o primeiro terço (de 0 a 3), considerado como baixo risco (cor verde);

- o segundo terço (de 4 a 6), como médio risco (cor amarela); e

- o terceiro terço (de 7 a 9), como alto risco (cor vermelha).

Acoplada a essa escala numérica têm-se as intervenções de enfermagem de acordo com a complicação encontrada, embasadas nos diagnósticos, resultados e intervenções de enfermagem: ligações entre: NANDA, NOC e NIC ${ }^{7}$.

Apesar da escala apresentar 14 complicações e seus riscos correspondentes, não necessariamente o paciente irá desenvolver todas, pois isso vai depender do seu histórico e das doenças associadas.

O Quadro 2 apresenta a escala das complicações, o questionário para classificação do nível dos riscos e as intervenções de enfermagem. 
Quadro 2. Instrumento para avaliação das complicações, riscos e intervenções de enfermagem na sala de recuperação anestésica.

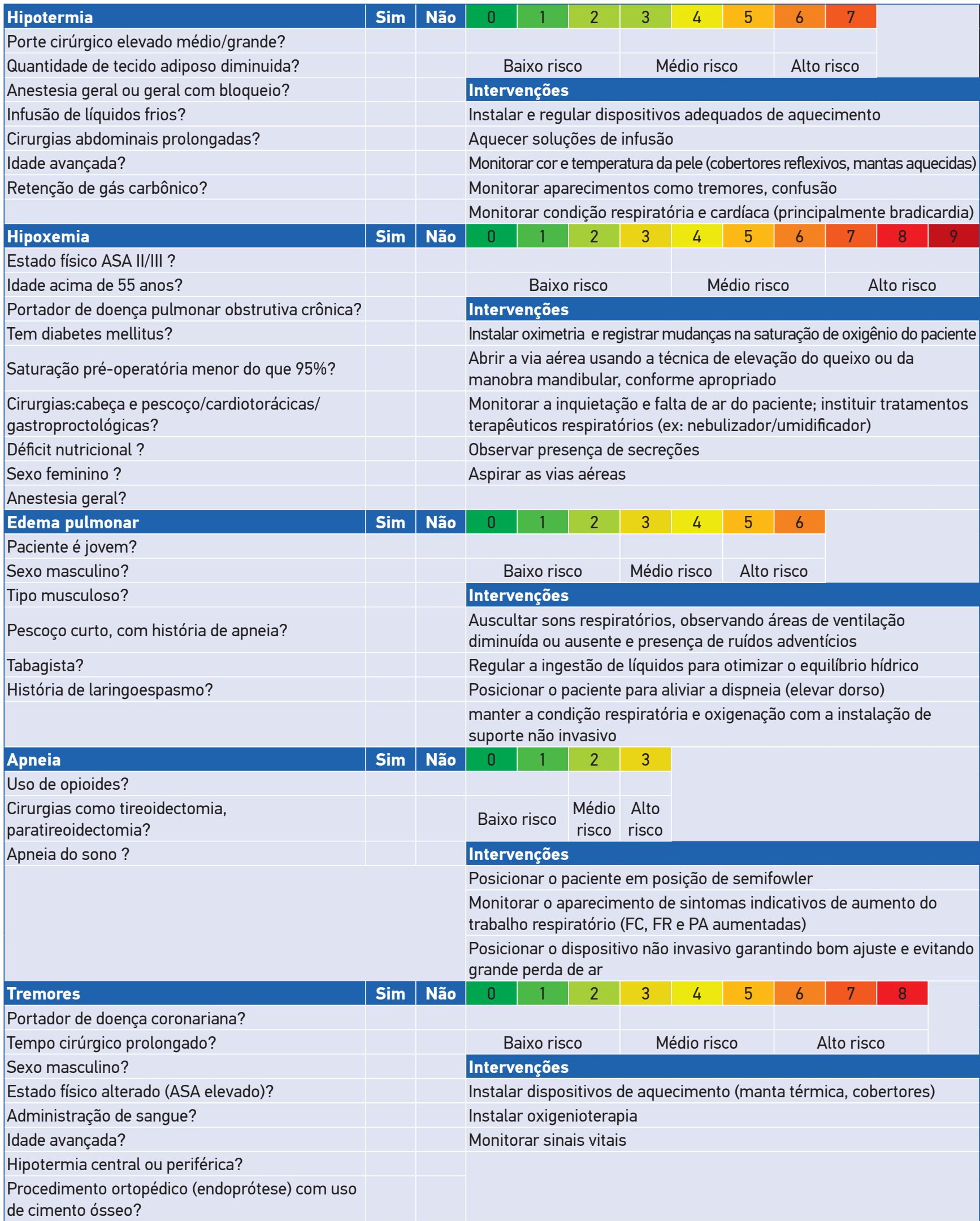


Quadro 2. Continuação.

\begin{tabular}{|c|c|c|c|c|c|c|c|c|c|c|c|}
\hline \multirow{2}{*}{$\begin{array}{l}\text { Náuseas e vômitos } \\
\text { E do sexo feminino? }\end{array}$} & Sim & Não & 0 & 1 & 2 & 3 & 4 & 5 & 6 & 7 & 8 \\
\hline & & & & & \multirow{2}{*}{\multicolumn{3}{|c|}{ Médio risco }} & \\
\hline Ausência de tabagismo? & & & & \multicolumn{3}{|c|}{ Baixo risco } & & & & & \\
\hline Antecedentes de NVPO? & & & \multicolumn{7}{|c|}{ Intervenções } & \multicolumn{2}{|c|}{ Alto risco } \\
\hline Náuseas e vômitos com movimento? & & & \multicolumn{9}{|c|}{ Posicionar para prevenir aspiração (elevar dorso) } \\
\hline Anestésicos inalatórios? & & & \multicolumn{9}{|c|}{ Oferecer saco para êmese } \\
\hline Uso do N2O na anestesia? & & & \multicolumn{9}{|c|}{ Promover higiene oral } \\
\hline Opioides intra e pós-operatórios? & & & \multicolumn{9}{|c|}{ Manter via aérea permeável } \\
\hline $\begin{array}{l}\text { Tempo cirúrgico superior a } 30 \text { minutos (aumenta } \\
\text { o risco em } 60 \% \text { )? }\end{array}$ & & & \multicolumn{9}{|c|}{ Identificar fatores de risco (medicações e procedimentos) } \\
\hline \multicolumn{12}{|l|}{$\begin{array}{l}\text { Cirurgias como: neurocirurgia, correção de } \\
\text { estrabismo, cirurgia plástica, laparotomia? }\end{array}$} \\
\hline \multirow[b]{2}{*}{ Retenção urinária } & \multirow[b]{2}{*}{ Sim } & & 0 & 1 & 2 & 3 & 4 & 5 & & & \\
\hline & & Não & Bai & risco & Médio & risco & Alto & isco & & & \\
\hline História prévia de retenção urinária? & & & Inte & nçõe & & & & & & & \\
\hline Recebeu opioides? & & & Prop & cionar & privacid & lade par & a elim & nação & & & \\
\hline Idade avançada? & & & $\begin{array}{l}\text { Estir } \\
\text { part }\end{array}$ & $\begin{array}{l}\text { lar } a b \\
\text { terna }\end{array}$ & $\begin{array}{l}\text { exiga re } \\
\text { da coxa, }\end{array}$ & $\begin{array}{l}\text { flexa, a } \\
\text { ou dei }\end{array}$ & $\begin{array}{l}\text { licand } \\
\text { rando }\end{array}$ & $\begin{array}{l}\text { frio } \\
\text { orrer }\end{array}$ & abd & & ssageando a \\
\hline $\begin{array}{l}\text { Excesso de fluidos administrados no período } \\
\text { transoperatório? }\end{array}$ & & & Real & rman & obra de & Credé s & houv & er ind & ç̧ão & & \\
\hline & & & reali & sond & agem de & alívio & uando & neces & ário & & \\
\hline Alterações do ritmo cardíaco & Sim & Não & 0 & 1 & 2 & 3 & 4 & 5 & 6 & 7 & 8 \\
\hline Anestesia geral? & & & & & & & & & & & \\
\hline Anestesia regional? & & & & ixo ris & & & dio ris & & & o ris & \\
\hline Extubação traqueal? & & & Inte & nçõe & & & & & & & \\
\hline Dor ao despertar? & & & Mon & rar alt & erações & da pres & זão sal & guíne & & & \\
\hline Náuseas e vômitos? & & & Obse & $\operatorname{ar} \sin$ & is e sint & omas c & débit & o carc & co dir & nuíd & \\
\hline Hipertermia? & & & Mon & rar a c & ondição & respira & ória & & & & \\
\hline Agitação ao despertar? & & & Mon & $\operatorname{tar} 0$ & bdome c & quando & da indi & cação & e pert & ão $d$ & ninuída \\
\hline Idade avançada? & & & $\begin{array}{l}\text { Prov } \\
\text { insti }\end{array}$ & $\begin{array}{l}\text { enciar } \\
\text { ção }\end{array}$ & terapia & antiar & ítmica & conf & & & \\
\hline & & & $\begin{array}{l}\text { reali } \\
\text { (enz }\end{array}$ & $\begin{array}{l}\text { colet } \\
\text { as car }\end{array}$ & $\begin{array}{l}\text { a de exa } \\
\text { díacas) }\end{array}$ & mes lak & orator & ais e & ompa & & ores \\
\hline Hipertensão arterial sistêmica & Sim & Não & 0 & 1 & 2 & 3 & 4 & 5 & 6 & & \\
\hline Idade avançada? & & & & & & & & & & & \\
\hline Dor? & & & & ixo ris & & Médio & risco & Altc & isco & & \\
\hline Distensão da bexiga? & & & Inte & nçõe & & & & & & & \\
\hline Tempo cirúrgico prolongado? & & & Mon & rar a c & corrênci & a de cia & nose $c$ & entral & perif & & \\
\hline Curativos oclusivos? & & & Mon & ar e c & ontrolar & a dor & & & & & \\
\hline Associação de fármacos? & & & Adm & strar & liurético & s, quan & lo ade & quado & & & \\
\hline & & & $\begin{array}{l}\text { mon } \\
\text { (hen }\end{array}$ & $\begin{array}{l}\text { rar re } \\
\text { ócrito }\end{array}$ & $\begin{array}{l}\text { sultados } \\
\text { sódio, c }\end{array}$ & $\begin{array}{l}\text { laborat } \\
\text { smola }\end{array}$ & $\begin{array}{l}\text { oriais r } \\
\text { idade }\end{array}$ & $\begin{array}{l}\text { eleva } \\
\text { urinár }\end{array}$ & es à & & e líquidos \\
\hline Hipotensão & Sim & Não & 0 & 1 & 2 & 3 & & & & & \\
\hline Bloqueios anestésicos? & & & & & & & & & & & \\
\hline Perda sanguínea? & & & Bai & risco & $\begin{array}{c}\text { Médio } \\
\text { risco }\end{array}$ & $\begin{array}{l}\text { Alto } \\
\text { risco }\end{array}$ & & & & & \\
\hline Ação das drogas anestésicas? & & & Inte & nçõe & & & & & & & \\
\hline & & & posi & nar 0 & vaciente & em pos & ção de & tren & lenbu & & \\
\hline & & & mon & rar pe & rda sang & uínea & & & & & \\
\hline & & & adm & trar $r$ & nedicaçã & confo & rme pr & escriç & & & \\
\hline
\end{tabular}


Quadro 2. Continuação.

\begin{tabular}{|c|c|c|c|c|c|c|c|c|}
\hline Obstrução e depressão respiratória & Sim & Não & 0 & 1 & 2 & 3 & 4 & 5 \\
\hline \multicolumn{9}{|l|}{ Queda da língua? } \\
\hline Excesso de saliva? & & & \multicolumn{2}{|c|}{ Baixo risco } & \multicolumn{2}{|c|}{ Médio risco } & \multicolumn{2}{|c|}{ Alto risco } \\
\hline Sangue? & & & \multicolumn{6}{|c|}{ Intervenções } \\
\hline Vômitos? & & & \multicolumn{6}{|c|}{$\begin{array}{l}\text { Abrir a via aérea usando a técnica de elevação do queixo ou da } \\
\text { manobra mandibular, conforme apropriado }\end{array}$} \\
\hline \multirow[t]{5}{*}{ Uso de opioides? } & & & \multicolumn{6}{|c|}{$\begin{array}{l}\text { Colocar o paciente deitado de lado, conforme indicado para evitar } \\
\text { aspiração }\end{array}$} \\
\hline & & & \multicolumn{6}{|c|}{$\begin{array}{l}\text { registrar movimentos torácicos observando a existência de simetria, } \\
\text { uso de músculos acessórios e retrações de músculos }\end{array}$} \\
\hline & & & \multicolumn{6}{|c|}{ supraclaviculares e intercostais } \\
\hline & & & \multicolumn{6}{|c|}{$\begin{array}{l}\text { monitorar a ocorrência de respirações ruidosas, como sibilos } \\
\text { esganiçados e roncos e dispneia }\end{array}$} \\
\hline & & & \multicolumn{6}{|c|}{ determinar necessidade de aspiração } \\
\hline Sangramento & Sim & Não & 0 & 1 & 2 & 3 & & \\
\hline \multicolumn{9}{|l|}{ Coagulopatias? } \\
\hline Problemas de hemostasia? & & & \multicolumn{2}{|c|}{ Baixo risco } & $\begin{array}{c}\text { Médio } \\
\text { risco }\end{array}$ & $\begin{array}{l}\text { Alto } \\
\text { risco }\end{array}$ & & \\
\hline \multirow[t]{8}{*}{ Cirurgias abdominais? } & & & \multicolumn{6}{|c|}{ Intervenções } \\
\hline & & & \multicolumn{6}{|c|}{ monitorar sinais de sangramento } \\
\hline & & & \multicolumn{6}{|c|}{ conter sangramento ativo se possível, aplicar curativo compressivo } \\
\hline & & & \multicolumn{6}{|c|}{ monitorar sinais vitais, estado mental e débito urinário } \\
\hline & & & \multicolumn{6}{|c|}{ monitorar a contagem de plaquetas, inclusive exames de coagulação } \\
\hline & & & \multicolumn{6}{|c|}{ preparar material para entubação } \\
\hline & & & \multicolumn{6}{|c|}{ providenciar bolsas de hemocomponentes para transfusão } \\
\hline & & & \multicolumn{6}{|c|}{ solicitar reserva de sala cirúrgica no caso de uma reoperação } \\
\hline Dor & Sim & Não & 0 & 1 & 2 & 3 & & \\
\hline \multicolumn{9}{|l|}{ Curativos oclusivos? } \\
\hline Ansiedade? & & & \multicolumn{2}{|c|}{ Baixo risco } & $\begin{array}{c}\text { Médio } \\
\text { risco }\end{array}$ & $\begin{array}{l}\text { Alto } \\
\text { risco }\end{array}$ & & \\
\hline Lesão tecidual média/grande? & & & Inte & ações & & & & \\
\hline & & & aplic & ão de c & alor/frio & o mass & gem & \\
\hline & & & aplic & ão do t & oque ter & rapêut & & \\
\hline & & & mus & terapia & para re & elaxar & & \\
\hline & & & conf & o no pc & siciona & amento & & \\
\hline & & & cont & e da ná & Iusea & & & \\
\hline & & & assi & ncia à a & analgesi & ia cont & olada & lo paciente \\
\hline & & & cont & e dos s & inais vit & tais (re & piraç & quando uso de opioides \\
\hline Posicionamento cirúrgico & Sim & Não & 0 & 1 & 2 & 3 & 4 & 5 \\
\hline Desnutrição? & & & & & & & & \\
\hline Diabetes mellitus? & & & & risco & Médio & risco & & isco \\
\hline Pontos ósseos? & & & Inte & ações & & & & \\
\hline Hiperextensão? & & & Posi & nar op & aciente & consic & rand & alinhamento correto do corpo \\
\hline $\begin{array}{l}\text { Maior abdução dos MMSS causando danos do } \\
\text { plexo braquial? }\end{array}$ & & & Mini & zar o at & rito e o & cisalh & ment & o posicionar e virar o paciente \\
\hline & & & $\begin{array}{l}\text { verif } \\
\text { cura }\end{array}$ & $\begin{array}{l}\text { ra inte } \\
\text { os se n }\end{array}$ & $\begin{array}{l}\text { gridade } \\
\text { ecessár }\end{array}$ & $\begin{array}{l}\text { da pe } \\
\text { rio }\end{array}$ & ; rea & a aplicação de cremes e realizar \\
\hline
\end{tabular}

ASA: American Society of Anesthesiologists; NVPO: náuseas e vômitos pós-operatórios; MMSS: membros superiores; FC: frequência cardíaca; FR: frequência respiratória; PA: pressão arterial. 


\section{DISCUSSÃO}

A revisão da literatura identificou a maioria dos artigos como delineamentos observacionais transversais, relatos de casos e revisões. Com base no referencial metodológico adotado, essas categorias de estudo se enquadram em níveis de evidências IV e $\mathrm{V}^{6}$.

Apesar da fragilidade dos estudos, eles possuem conceitos e informações importantes que respondem à questão norteadora da revisão e serviram de base para a construção do instrumento, após avaliação estatística.

Os principais temas identificados por meio da análise crítica dos artigos e suas implicações são apresentados e discutidos na sequência.

\section{Hipotermia}

Nos três artigos, a anestesia geral foi a técnica mais utilizada, seguida da anestesia combinada (geral mais bloqueios do neuroeixo), as quais representam situações de maior risco de apresentar hipotermia perioperatória não intencional, pois a anestesia regional diminui o limiar de vasoconstrição cutânea e quando associada à geral tem seu efeito somatizado ${ }^{8-10}$.

A hipotermia interfere no ritmo e na condução do coração com o aparecimento de disritmias, e na incidência de infecção do sítio cirúrgico ${ }^{8-10}$.

No que se refere ao tempo cirúrgico, a maior frequência de hipotermia leve e moderada encontra-se no tempo cirúrgico mínimo de 61 minutos e máximo de 240 minutos $^{9}$. A ausência de métodos preventivos de hipotermia (como aquecedores) em sala operatória foi a causa de temperatura abaixo de $35^{\circ} \mathrm{C}$ nos pacientes durante o período transoperatório. E quando admitidos na SRA, esses pacientes se mantiveram hipotérmicos ainda por 30 minutos $^{8}$.

A quantidade de tecido adiposo do paciente e o uso concomitante de medicações anestésicas podem alterar o gradiente de temperatura entre os compartimentos central e periférico, levando a uma hipotermia ${ }^{10}$.

Tendo como principais riscos para hipotermia:

- porte cirúrgico médio e grande, maior risco de hipotermia;

- quantidade de tecido adiposo;

- anestesia geral ou geral associada com bloqueio;

- infusão de líquidos não aquecidos;

- tempo cirúrgico prolongado;

- cirurgias abdominais nas quais ocorre maior perda de calor;

- idade avançada; e

- retenção de gás carbônico.

\section{Hipoxemia}

Em dois trabalhos, os pacientes que receberam anestesia geral, devido ao efeito residual das drogas anestésicas, foram os que apresentaram, em maior número, hipoxemia no pós-operatório ${ }^{11,12}$.

Outra variável que pode levar o paciente a desenvolver hipoxemia na SRA e a classificação ASA da American Society of Anesthesiologists (ASA) ${ }^{13}$, a qual classifica os pacientes mediante a ausência ou presença de moléstias associadas ao problema cirúrgico. Quanto mais alta a classificação na escala (1 a 6), maior será a possibilidade do paciente desenvolver hipoxemia.

As cirurgias cardiotorácicas, gastroproctológicas e de cabeça e pescoço, em pacientes oncológicos, associadas à debilidade física e nutricional, são fatores que podem levar o paciente à hipoxemia ${ }^{11}$.

Pacientes idosos possuem déficit nutricional e diminuição da reserva respiratória, e estão mais expostos ao risco de manifestarem hipoxemia na SRA ${ }^{12}$.

Variáveis que podem levar o paciente a indicar hipoxemia:

- estado físico ASA II e III;

- idade acima de 55 anos;

- DPOC;

- diabetes mellitus;

- $\mathrm{SpO}_{2}$ pré-operatório menor que 95\%;

- déficit nutricional;

- pacientes do sexo feminino; e

- anestesia geral.

\section{Apneia e edema agudo de pulmão}

Pacientes que apresentam laringoespasmos na SRA podem evoluir para edema agudo de pulmão ${ }^{14}$.

O uso da oxigenioterapia é indicado principalmente após a anestesia geral. E em procedimentos de cabeça e no pescoço, a cirurgia de tireoidectomia é um fator de risco para apneia na SRA ${ }^{15}$.

Pacientes que apresentam laringoespasmos na SRA podem evoluir para edema agudo de pulmão ${ }^{14}$.

São fatores de riscos para edema agudo de pulmão:

- maior frequência em pacientes jovens;

- sexo masculino; tipo musculoso;

- pescoço curto;

- com história de apneia;

- tabagista;

- laringoespasmo. 
São fatores de risco para apneia:

- uso de opioides;

- cirurgias como tireoidectomia e paratireoidectomia;

- apneia do sono.

\section{Tremores, náuseas, vômitos e retenção urinária}

Os fatores desencadeantes de tremores na SRA acometem pacientes com doença coronariana ${ }^{16}$, tendo como fatores de risco:

- doença coronariana devido ao aumento do consumo de oxigênio;

- intervenções cirúrgicas com tempo prolongado;

- sexo masculino;

- estado físico alterado (ASA elevado);

- administração de sangue;

- idade avançada;

- hipotermia central ou periférica;

- procedimento ortopédico (endoprótese) com uso de cimento ósseo: existe um risco para tremores no pós-operatório. Uma explicação seria a liberação de citosinas estimulada pelo uso do cimento ósseo ${ }^{16}$.

Náuseas e vômitos pós-operatórios (NVPO) podem ser desencadeados com maior frequência por um grupo de pacientes. Após revisão exaustiva da literatura, foi elaborada a definição desse grupo de risco, sendo os pacientes com maior incidência de riscos ${ }^{17}$ :

- sexo feminino;

- ausência de tabagismo;

- antecedentes de náuseas e vômitos

- náuseas e vômitos com movimento;

- anestésicos inalatórios;

- oxido nitroso $\left(\mathrm{N}_{2} \mathrm{O}\right)$;

- opioides intra e pós-operatórios;

- tempo cirúrgico superior a 30 minutos (aumenta o risco em $60 \%$ );

- neurocirurgia, cirurgias para correção de estrabismo, cirurgia plástica e laparotomia ${ }^{17}$.

Em relação ao débito urinário, foram considerados fatores preditivos independentes na SRA as cirurgias sobre os membros inferiores e o volume urinário igual ou superior a $360 \mathrm{~mL}$ no pós-operatório, assim como a idade do paciente e a administração de opioides também ${ }^{18}$.

Fatores de risco:

- cirurgias anorretais;

- herniorrafias inguinais;

- história prévia de retenção urinária;
- opioides;

- idade avançada;

- excesso de fluidos administrados no período transoperatório.

\section{Grau de dependência de cuidados e disritmias cardíacas}

Na SRA, os pacientes com cuidados intensivos apresentam necessidades de monitorização hemodinâmica e até invasiva, suporte ventilatório, medida do débito urinário e drenagens de sondas e drenos, entre outras atividades, dificultando a rotatividade dos leitos ${ }^{1}$.

Os pacientes portadores de doença cardiovascular prévia e com idade acima de 60 anos estão mais propensos a apresentar disritmias na SRA ${ }^{19}$.

Taquicardia sinusal é a mais frequente das alterações cardiovasculares na SRA, seguida de bradicardia sinusal, extrassístoles ventriculares e atriais ${ }^{19}$.

Os fatores de riscos para taquicardia sinusal são:

- anestesia geral (droga como sulfato de atropina);

- extubação traqueal;

- dor ao despertar;

- náuseas e vômitos; e

- hipertermia.

O fator de risco para bradicardia sinusal é:

- a anestesia geral.

Para extrassístoles ventriculares e extrassístoles atriais:

- extubação traqueal e

- agitação ao despertar.

Com a variação de um ou dois riscos apenas não é possível a confecção da escala, pois a mesma necessita de pelo menos três riscos para classificação em baixo, médio e alto. Então optou-se por elaborar uma escala que contemple as complicações e os riscos que estão envolvidos em relação às alterações do ritmo cardíaco.

\section{Complicações gerais, com idosos e intervenções na SRA}

As principais complicações relatadas foram: hipotensão devido ao bloqueio anestésico, depressão respiratória, na maioria das vezes causada pela ação das drogas depressoras do bloqueio neuromuscular ${ }^{20}$. 
Em outro trabalho foram destacadas as seguintes complicações: dor, agitação, ansiedade, hipotensão, tremores e calafrios, náuseas, vômitos, sangramentos e hipoxemia. A anestesia mais realizada foi a geral e a comorbidade mais frequente foi a hipertensão arterial sistêmica (HAS) ${ }^{2}$.

Na população de idosos, as complicações mais comuns na SRA foram: hipotermia seguida da dor, aumento da pressão arterial, náuseas e vômitos, dispneia, taquipneia e bradicardia ${ }^{3}$.

Nos pacientes idosos o tempo cirúrgico é outro fator de risco. Quanto maior ele for, maior a chance de desencadear eventos cardíacos e respiratórios ${ }^{3,20}$.

A seguir os fatores de risco para as complicações:

- hipertensão arterial sistêmica: idade avançada; dor; distensão da bexiga; tempo cirúrgico prolongado; curativos oclusivos; associação de fármacos;

- hipotensão: bloqueios anestésicos; perda sanguínea; ação das drogas anestésicas;

- bradicardia: anestesia regional; sangramentos;

- taquicardia: agitação; idade avançada (mais de 60 anos);

- obstrução respiratória: queda da língua; saliva; sangue e vômitos;

- depressão respiratória: medicações de opioides;

- sangramento: coagulopatias; problemas de hemostasias; cirurgias abdominais;

- dor: curativos oclusivos; ansiedade; trauma.

\section{Posicionamento cirúrgico}

A posição cirúrgica pode ocasionar efeitos gravitacionais no sistema cardiorrespiratório do paciente e tem como fatores de risco ${ }^{3}$ :

- desnutrição;

- diabetes mellitus;

- pontos ósseos;

- hiperextensão;
- maior abdução dos membros superiores causando danos do plexo braquial.

\section{Limitações}

Esta revisão integrativa apresentou algumas limitações, como o número reduzido de produções no que se refere à assistência de enfermagem prestada na SRA.

Os artigos internacionais escritos por enfermeiros não são a nossa realidade, pois a maioria dos trabalhos está voltada para ação das drogas e não para assistência de enfermagem prestada.

Os artigos e as publicações trazem referenciais didáticos baseados em tipos de estudos com níveis de evidência fraca, níveis V e VI.

\section{CONCLUSÃO}

O levantamento realizado na literatura mostrou as seguintes complicações na SRA: hipotermia, hipoxemia, edema pulmonar; apneia, tremores, náuseas e vômitos; retenção urinária, alterações do ritmo cardíaco, hipertensão arterial; hipotensão, depressão respiratória; sangramento; dor; e o próprio posicionamento cirúrgico como fator desencadeante de complicações no pós-operatório imediato.

Após as complicações descritas foram levantados os riscos, que serviram de base para a construção do questionário, com perguntas e respostas - sim (vale 1) e não (vale 0 ) no qual a somatória dos pontos classifica o nível do risco em baixo, médio e alto para o paciente desenvolver a complicação.

A escala das complicações, riscos e intervenções de enfermagem, aqui proposta, deverá ser validada na prática. Espera-se, com isso, que os enfermeiros, com o levantamento precoce dos riscos, venham a prevenir as complicações e realizar uma assistência de enfermagem mais segura.

\section{REFERÊNCIAS}

1. Lima LB de, Borges D, CostaS da, Rabelo ER. Classificação de pacientes segundo o grau de dependência dos cuidados de enfermagem e a gravidade em unidade de recuperação pós-anestésica. Rev. Latino-Am Enfermagem [internet]. 2010 [cited on 2015 maio 20]; 18(5). Available from: http://dx.doi.org/10.1590/S0104-11692010000500007

2. Popov DCS, Peniche ACG. As intervenções do enfermeiro e as complicações em sala de recuperação pós-anestésica. Rev Escola
Enferm USP [internet]. 2009 [cited on 2015 jun 24];43(4):953-61. Available from: http://producao.usp.br/handle/BDPI/4084

3. Mendoza IYQ, Peniche ACG. Factores de riesgo para complicaciones en el periodo de recuperación post anestésica en el paciente anciano. Invest Educ Enferm [internet]. 2010 [cited on 2015 jul 24];28(3):355-62. Available from: http://www.redalyc.org/articulo. oa? id $=105215721006$ 
4. Aldret JAD. Apostanesthetic recovery score. Anesth Anag. 1970;49:924-34.

5. Mendes KDS, Silveira RCCP, Galvão CM. Revisão Integrativa: Método de pesquisa para a incorporação de evidências na saúde e na enfermagem. Rev Texto Contexto Enferm [internet]. 2008 [cited on 2015 out 10];17(4):758-64. Available from: http://producao.usp.br/ handle/BDPI/3509

6. Stetler CB, Morsi D, Rucki S, Broughton S, Corrigan B, Fitzgerald J, et al. Utilization-focused integrative reviews in a nursing service. Appl Nurs Res. 1998;11(4):195-206.

7. Johnson M, Moorhead S, Bulecheck G, Butcher H, Maas M, Swanson E. Diagnósticos, resultados e intervenções de enfermagem: ligações entre NANDA, NOC e NIC. 3a ed. Rio de Janeiro: Elsevier; 2012.

8. Mattia AL, Barbosa MH, Rocha AM, Farias HL, Santos CA, Santos DM. Hipotermia em pacientes no período perioperatório. Rev Escola Enferm USP [internet]. fev. 2012 [cited on 2015 maio 16];46(1):60-6. Available from: http://dx.doi.org/10.1590/ S0080-62342012000100008

9. Amante LN, Slomochenski LA, Texeira MGPN, Bertoncello KCG. Ocorrência de hipotermia não planejada em sala de recuperação anestésica. UNOPAR Cient Ciênc Biológicas Saúde. 2012 [cited on 2015 ago 12];14(4):211-5. Available from: http://www.pgsskroton. com.br/seer/index.php/JHealthSci/article/view/8

10. Zappelini CEM, Sakae TM, Bianchini N, Brum SPB. Avaliação de hipotermia na sala de recuperação pós-anestésica em pacientes submetidos a cirurgias abdominais com duração maior de duas horas. Arq Catarin Med. 2008 [cited on 2015 mar 15];37(2):25-31. Available from: http://bases.bireme.br/cgi-bin/wxislind.exe/iah/online/?IsisScript=iah/iah.xis\&base=LILACS\&lang=p\&nextAction=lnk\&exprSearch $=499736$ \&indexSearch $=$ ID

11. Marcondes G, Soeiro FS, Ferreira EA, Udelsmann A. Transporte de pacientes sem oxigenioterapia para a sala de recuperação pósanestésica: repercussões na saturação de oxigênio e fatores de risco associados à hipoxemia. Rev Bras Anestesiol [internet]. 2006 [cited on 2015 abr 16];56(4):352-61. Available from: http://dx.doi. org/10.1590/S0034-70942006000400003

12. Oliveira Filho GR, Garcia JHS, Ghellar MR, Nicolodi MA, Boso AL, Dal Mago AJ. Fatores associados com a ocorrência de hipoxemia no período pós-anestésico imediato. Rev Bras Anestesiol [internet]. 2001 [cited on 2015 ago 12];51(3): 185-95. Available from: http:// dx.doi.org/10.1590/S0034-70942001000300001

13. American Society of Anesthesiologists (ASA). ASA Physical Status Classification System. ASA [internet]. 2014. [cited on 2015 jul 02]. Available from: https://www.asahq.org/resources/clinical-information/ asa-physical-status-classification-system

14. Bisinotto FMB, Cardoso RP, Abud TMV. Edema agudo pulmonar associado à obstrução das vias aéreas: relato de caso. Rev Bras Anestesiol [internet]. 2008 [cited on 2015 jul 02];58(2):165-71. Available from: http://www.scielo.br/scielo. php?script=sci_arttext\&pid=S0034-70942008000200009\&lng=en

15. Rezende JM. Apnéia na sala de recuperação pós-anestésica: relato de caso. Rev Bras Anestesiol. 2003;53(3):377-81.

16. Albergaria VF, Lorentz MN, Lima FA de. Tremores intra e pósoperatório: prevenção e tratamento farmacológico. Rev Bras Anestesiol [internet]. 2007 [cited on 2015 set 17];57(4):431-44. Available from: http://dx.doi.org/10.1590/S0034-70942007000400012

17. Lages N, Fonseca C, Neves A, Landeiro N, Abelha FJ. Náuseas e vômitos no pós-operatório: uma revisão do "pequeno-grande" problema. Rev Bras Anestesiol [internet]. 2005 [cited on 2015 ago 19];55(5):575-85. Available from: http://dx.doi.org/10.1590/ S0034-70942005000500013

18. Mago AD, Helayel P, Bianchini E, Kozuki H, Oliveira FGR de. Prevalência e fatores preditivos de retenção urinária diagnosticada por ultrassonografia no período pós-anestésico imediato. Rev Bras Anestesiol [internet]. 2010 [cited on 2015 abr 16];60(4):387-90. Available from: http://dx.doi.org/10.1590/\$0034-70942010000400005

19. Nascimento Júnior P, Castiglia YMM. Disritmias cardíacas per e pós-operatórias em pacientes sem doença cardiovascular. Rev Bras Anestesiol [internet]. 2000 [cited on 2015 jun 11];50(5):3506. Available from: http://bases.bireme.br/cgi-bin/wxislind.exe/iah/ online/?lsisScript=iah/iah. $x i s \& s r c=$ google $\&$ base $=$ LILACS\&lang=p\&nextAction=lnk\&exprSearch=277494\&indexSearch=ID

20. Eltringham RJ. Complications in the recovery room. J Royal Society Med [internet]. 1979 [cited on 2015 set 05];72(4):278-80. Available from: https://www.ncbi.nlm.nih.gov/pmc/articles/PMC1437023/ 